\title{
Role of Cell-Origin Profiling Using Immunohistochemistry to Predict the Survival of Patients with Diffuse Large B-Cell Lymphoma in Indonesia
}

\author{
Hermawan Istiadi,* Udadi Sadhana," Dik Puspasari, $†$ Ika Pawitra Miranti,* Vega Karlowee," Devia Eka Listiana $\uparrow$ \\ and Awal Prasetyo* \\ *Anatomical Pathology Department, Faculty of Medicine, Diponegoro University, Semarang 50244, Indonesia, and †Anatomical \\ Pathology Laboratory, Kariadi General Hospital, Semarang 50244, Indonesia
}

\begin{abstract}
Background Diffuse large B-cell lymphoma (DLBCL) is the most common type of non-Hodgkin lymphoma in Asia and Indonesia. DLBCL could be further classified according to cell of origin as the germinal center B-cell (GCB) subtype or the non-germinal center B-cell (nonGCB) subtypes; of these, the non-GCB subtype usually has poorer prognosis. The purpose of this study is to determine the relationship between the cell-origin subtype and 3-year overall survival of patients with DLBCL at Kariadi General Hospital Semarang.

Methods This research represents an observational analytical study of 36 patients with DLBCL who visited Kariadi General Hospital between January and August 2017. Data on age of diagnosis, tumor location, disease stage, and 3-year overall survival were collected. DLBCL subtype was determined via immunohistochemical examination of CD10, BCL6, and MUM1 protein expression. Data analyses, including the chi squared test and Kaplan-Meier curves, were conducted.
\end{abstract} Results The study population included 18 patients with GCB-subtype DLBCL and 18 patients with nonGCB-subtype DLBCL. No significant difference $(P=$ $0.171)$ between disease stage and cell-origin subtype was noted between groups. Patients with the nonGCB subtype had a 3-year overall survival that was significantly worse than that of patients with the GCB subtype $(P=0.026)$. Moreover, the 3 -year survival rate of patients with the non-GCB subtype of the disease was $38.9 \%$ while that of patients with the GCB subtype was $77.8 \%$. Patients with advanced stages of DLBCL also had a 3-year overall survival that was significantly worse than those of patients with early stages of the disease $(P<0.001)$, with the 3 -year survival rate of patients with advanced stage was $14.3 \%$.

Conclusion Patients with non-GCB-subtype DLBCL or advanced stages of the disease have a lower 3-year overall survival rate and poorer prognosis compared with those with other subtypes or earlier stages of the disease.

Key words diffuse; Indonesia; large B-cell; lymphoma; survival rate
Lymphoma, a malignancy arising from lymphoid tissue, can be divided into two main groups: Hodgkin lymphoma (HL) and non-Hodgkin lymphoma (NHL). Over $85 \%$ of all lymphoma cases in the world are NHL, and more than $90 \%$ of these cases could be classified as mature B cells NHL. ${ }^{1,2}$ In Indonesia, NHL is the seventh most common cancer. The most common type of NHL in Asia, including Indonesia, is diffuse large B-cell lymphoma (DLBCL). Approximately $64 \%$ of all patients with DLBCL are found in advanced stages of the disease (Ann Arbor stages III and IV). ${ }^{1-3}$

DLBCL is an aggressive B-cell NHL characterized histologically by a diffuse pattern of malignant lymphoid cells expressing various B cell markers, including CD20, CD79a, CD19, CD22, PAX5, BOB1, and OCT2; the cells also have a high $\mathrm{Ki}-67$ proliferation index. $^{2-4}$ DLBCL can be classified into two subtypes according to the molecular profile of the original cells, namely germinal center B-cell type (GCB) and non-germinal center B-cell type (non-GCB), by immunohistochemical examination of CD10, BCL6 and MUM1. 2, 5, 6 In general, GCB-subtype DLBCL has better prognosis and overall survival compared with the non-GCB subtype. ${ }^{2,7}$

Case data of lymphoid tissue malignancies in Indonesia are neither well developed nor widely available. The accurate diagnosis of DLBCL cases in Indonesia is also complicated by the general lack of immunohistochemiscal examination. Achieving a complete diagnosis of DLBCL, including its molecular subtype, is very important to support immunotherapy efforts and predict the prognosis of affected patient. This study aims to determine the prevalence of DLBCL patients based on its cell of origin immune-profile and its correlation with clinical features and 3-year overall survival, in Kariadi General Hospital Semarang.

Corresponding author: Hermawan Istiadi, MD

hermawanistiadi@fk.undip.ac.id

Received 2020 August 21

Accepted 2021 April 26

Online published 2021 May 20

Abbreviations: DLBCL, Diffuse Large B-Cell Lymphoma; GCB, Germinal Center B-cell; HL, Hodgkin's lymphoma; NHL, nonHodgkin's lymphoma 


\section{MATERIALS AND METHODS Patients and methods}

This research represents an observational analytical study with a retrospective cohort design. A total of 36 patients who had been diagnosed with DLBCL based on histopathological and immunohistochemical examinations, including diffuse CD20 and high Ki-67 expression, by the Anatomical Pathology Laboratory of Kariadi General Hospital, Semarang, which is the top referral hospital in Central Java, Indonesia, between January and August 2017 were recruited to this study. The patients were followed-up for 3 years after diagnosis and treatment to determine their 3-year overall survival. The study protocol was approved by the Ethics Committee of the Faculty of Medicine, Diponegoro University (Authorization number: 106/EC/FK-RSDK/ III/2018).

\section{Immunohistochemistry examination method}

DLBCL specimens from all patients were prepared into paraffin blocks and sectioned into approximately $4 \mu \mathrm{m}$ thick slices by using a microtome. One section each was used for CD10 staining, BCL6 staining, and MUM1 staining. Paraffin sections were deparaffinized using xylene, rehydrated using ethanol and washed in phosphate buffered saline ( $\mathrm{pH}$ 7.4).

Antigen retrieval using Tris-EDTA buffer (PH 9) was conducted in a microwave for $10 \mathrm{~min}$ at $750 \mathrm{~W}$ and $15 \mathrm{~min}$ at $350 \mathrm{~W}$ and slide staining used monoclonal antibodies of CD10, BCL6 and MUM1 (Leica Biosystem, Newcastle, UK) at 1: 100 dilution. All stained slides were examined at $100 \times$ and $400 \times$ magnification, and positivity was determined. CD10 expression was considered positive if immunostaining localized in the plasma membrane of malignant lymphoid cells was observed, BCL6 and MUM1 expression were considered positive if immunostaining localized in the nucleus was noted. According to the Hans algorithm, the germinal center B-cell (GCB) subtype was defined as CD10+ or BCL6+ and MUM1-, and all others (CD10- and BCL6- or CD10- and MUM1+) were defined as the non-GCB subtype. $^{8}$

\section{Statistical methods}

The clinical features of the patients, including age of diagnosis (age $>50$ years vs. age $<50$ years), tumor location (nodal, extra nodal), Ann Arbor tumor stage [early stage (stage I and II) vs. advanced stages (stage III and IV)], and cell-origin subtype based on immunohistochemical examination of CD10, BCL6 and MUM1 were collected. The overall survival of a patient was evaluated in terms of number of months they remained alive from diagnosis until August 2020. Data analysis was carried out using Statistical Package for Social Sciences (IBM SPSS statistic, version 21, IBM Chicago, IL). Correlations between variables were analyzed using the chi squared test with a significance level of $<0.05$, and survival analysis was conducted using KaplanMeier curves with the log-rank test.

\section{RESULTS}

\section{Clinicopathological features of the patients}

Thirty-six patient with DLBCL at Kariadi General Hospital were included in this study. Most (52.8\%) of the patients were diagnosed at an age of over 50 years, with a median age of diagnosis of 52 years. The majority of the patients had tumors at extra nodal locations (75\%). The most common location of extra nodal tumors included the gastrointestinal tract, liver, spleen, kidney, nasal cavity, mediastinum, tonsils, palpebra, conjunctiva, central nervous system, and femur. The most common locations of nodal tumors included the cervical, inguinal, axillary, and submandibular lymph nodes; the tumors could be observed singly or in multiple clusters. In terms of Ann Arbor staging, most of the patients had early-stage DLBCL (61.1\%; Table 1).

\section{Clinicopathological features according to cell- origin subtype}

In terms of cell-origin subtype, 18 patients (50\%) had the GCB subtype, while 18 patients $(50 \%)$ had the nonGCB subtype (Fig. 1). Three years after diagnosis and receiving R-CHOP treatment, 15 patients (41.7\%) died.

Among the patients with GCB-subtype DLBCL in this study, $55.5 \%$ were diagnosed at older than 50 years of age, $72.2 \%$ had tumors with extra nodal locations, and $72.2 \%$ were at early stages of the disease. Among patients with non-GCB subtype DLBCL, 50\% were diagnosed at older than 50 years of age, $77.8 \%$ had tumors with an extra nodal location, and $50 \%$ were at early stages of the disease. No significant difference and correlation between age of diagnosis, location, and stage with cell-origin subtype of DLBCL was observed (Table 2).

\section{Overall survival of patients}

After 3 years of follow-up, 15 patients died and 21 patients survived. Among the 15 patients who died, 4 had the GCB subtype and 11 had the non-GCB subtype. Therefore, at the end of the follow-up period, 14 patients with GCB-subtype DLBCL survived (77.8\%) and 7 patients with non-GCB-subtype DLBCL survived $(38.9 \%)$. The median survival of non-GCB patients was 6 months. 
Table 1. Clinicopathological features of DLBCL patients $(n=36)$

\begin{tabular}{|c|c|c|}
\hline & Frequency & Percentage \\
\hline Age of diagnosis & \multicolumn{2}{|c|}{$51.83 \pm 14.08$ years (median: 52 years, min: 22 years, max: 77 years) } \\
\hline \multicolumn{3}{|l|}{ Age category } \\
\hline$<50$ years & 17 & $47.20 \%$ \\
\hline$>50$ years & 19 & $52.80 \%$ \\
\hline \multicolumn{3}{|l|}{ Location } \\
\hline Nodal & 9 & $25 \%$ \\
\hline Extra nodal & 27 & $75 \%$ \\
\hline \multicolumn{3}{|l|}{ Stage } \\
\hline Early (I, II) & 22 & $61.10 \%$ \\
\hline Advanced (III, IV) & 14 & $38.90 \%$ \\
\hline \multicolumn{3}{|l|}{ Cell-origin subtype } \\
\hline GCB & 18 & $50 \%$ \\
\hline Non GCB & 18 & $50 \%$ \\
\hline \multicolumn{3}{|l|}{ 3-year overall survival } \\
\hline Alive & 21 & $58.30 \%$ \\
\hline Dead & 15 & $41.70 \%$ \\
\hline
\end{tabular}

In terms of disease stage, among the 15 patients who died during follow-up, 3 had early-stage DLBCL and 12 had advanced-stage DLBCL. Therefore, 18 patients with early-stage DLBCL survived (86.4\%) and 2 patients with advanced-stage DLBCL survived (14.3\%) until the end of follow up. The median survival time of patients with advanced-stage DLBCL was 5 months (Fig. 2).

\section{DISCUSSION}

In this study, 36 patients with DLBCL who visited Kariadi General Hospital Semarang between January 2017 and August 2017, were followed-up for the next 3 years to determine their 3-year overall survival and the clinical factors influencing survival, including age at diagnosis, tumor location (nodal/extra nodal), Ann Arbor tumor stage, and cell-origin subtype (GCB/nonGCB) by using immunohistochemical examination. Patients with DLBCL are usually diagnosed at an older age, with a median age in the sixth or seventh decade; however, some patients are diagnosed at a younger age. Several studies conducted in East Asia have reported that the median age of patients with DLBCL at the time of diagnosis is the fifth decade. ${ }^{9}, 10$ These previous findings coincide with the result of the present study, where the age of diagnosis of patients with DLBCL is approximately 51 years; the youngest patient was diagnosed at 22 years, and the oldest patient was diagnosed at 77 years. Patients with GCB-subtype DLBCL in this study were more likely to be diagnosed at age of $>50$ years, while those with non-GCB-subtype DLBCL were diagnosed at equal proportions of less than 50 years and more than 50 years. No significant difference between age of diagnosis and cell-origin subtype of DLBCL was noted in this study. This finding is in line with previous studies showing that patients with GCB-subtype DLBCL are more likely to be diagnosed at an older age than non-GCB patients; No significant difference and correlation between age of diagnosis with cell-origin subtype of DLBCL were noted. ${ }^{11-13}$

Nearly $50 \%$ of the patients with DLBCL were diagnosed with stage I or II (early stage) disease without a PET/CT scan. If a PET/CT scan was added as an examination, the prevalence of stages I and II DLBCL patients may be reduced. While approximately $64 \%$ of all DLBCL patients had stage III or IV disease, in other studies in Asia, DLBCL patients (52-60\%) were slightly more likely to be classified with stage I or II disease. ${ }^{14-16}$ In the present study, most patients with DLBCL at the Kariadi General Hospital had stage I or II (61.1\%) disease according to the results of the CT scan of the patient. Approximately $72.2 \%$ of the patients with GCB-subtype DLBCL in this study were diagnosed at stage I or II, while $50 \%$ of the patients with non-GCBsubtype DLBCL were diagnosed at stage I or II; the rest were diagnosed with stage III or IV disease. Therefore, no significant difference and correlation were found between disease stages and cell-origin subtype. Previous 

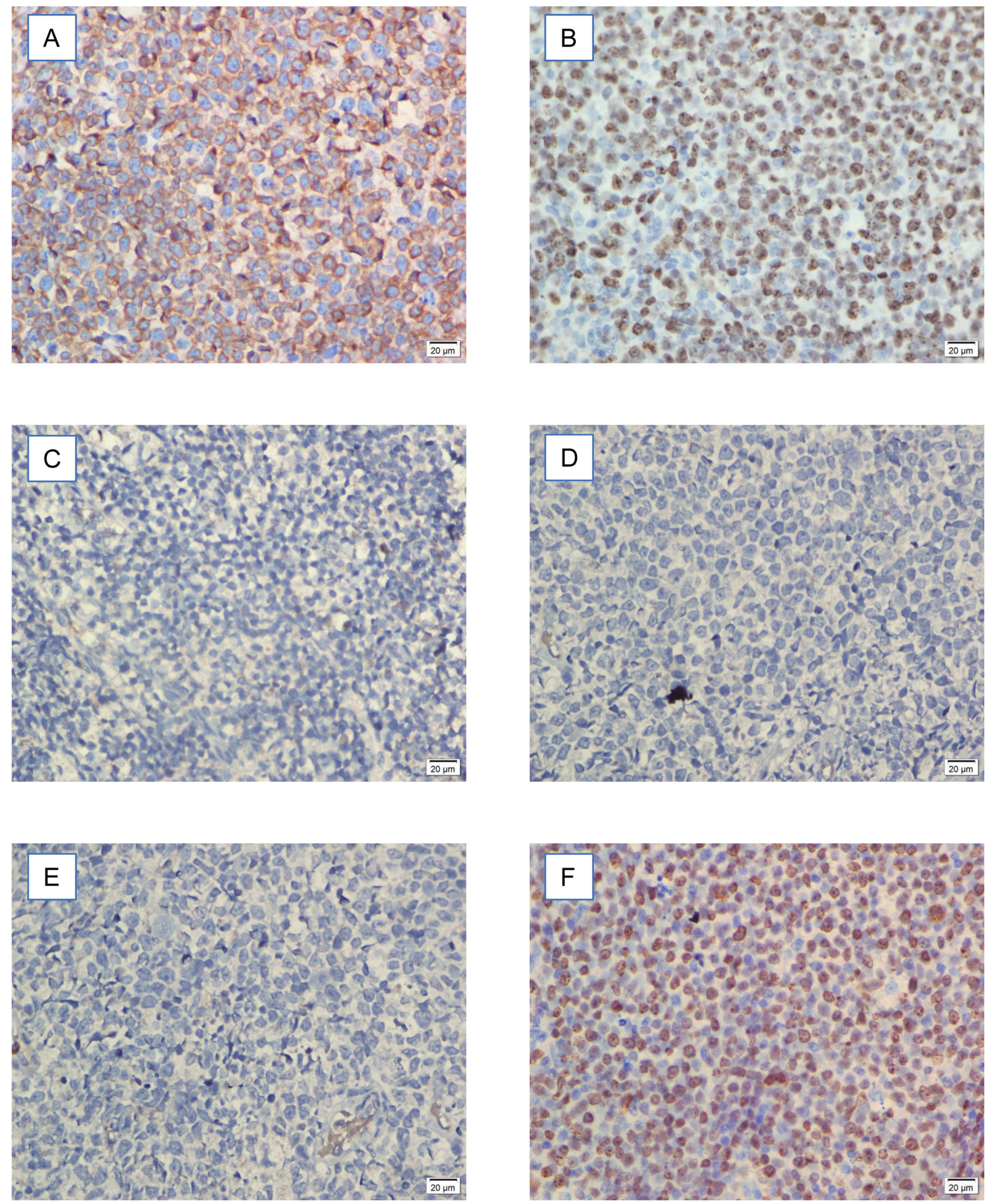

Fig. 1. A: CD10 positivity in GCB (germinal center B-cell) DLBCL (diffuse large B-cell lymphoma). B: BCL6 positivity in GCB DLBCL. C: MUM1 negativity in GCB DLBCL. D: CD10 negativity in non-GCB DLBCL. E: BCL6 negativity in non-GCB DLBCL. F: MUM1 positivity in non-GCB DLBCL. Bar $=20 \mu \mathrm{m}$. 
Table 2. Correlation of age of diagnosis, location, and stage with the cell-origin subtype of DLBCL

\begin{tabular}{lccc}
\hline & GCB & Non GCB & $P$ \\
\hline Age of diagnosis & & & \\
$<50$ years & $8(44.5 \%)$ & $9(50 \%)$ & 0.738 \\
$>50$ years & $10(55.5 \%)$ & $9(50 \%)$ & 0.7 \\
\hline Location & & & $4(22.2 \%)$ \\
$\quad$ Nodal & $5(27.8 \%)$ & $14(77.8 \%)$ & 0.171 \\
Extra nodal & $13(72.2 \%)$ & & $9(50 \%)$ \\
\hline Stage & & $9(50 \%)$ & \\
Early (I, II) & $13(72.2 \%)$ & $5(27.8 \%)$ & \\
Advanced (III, IV) & & \\
\hline
\end{tabular}

A

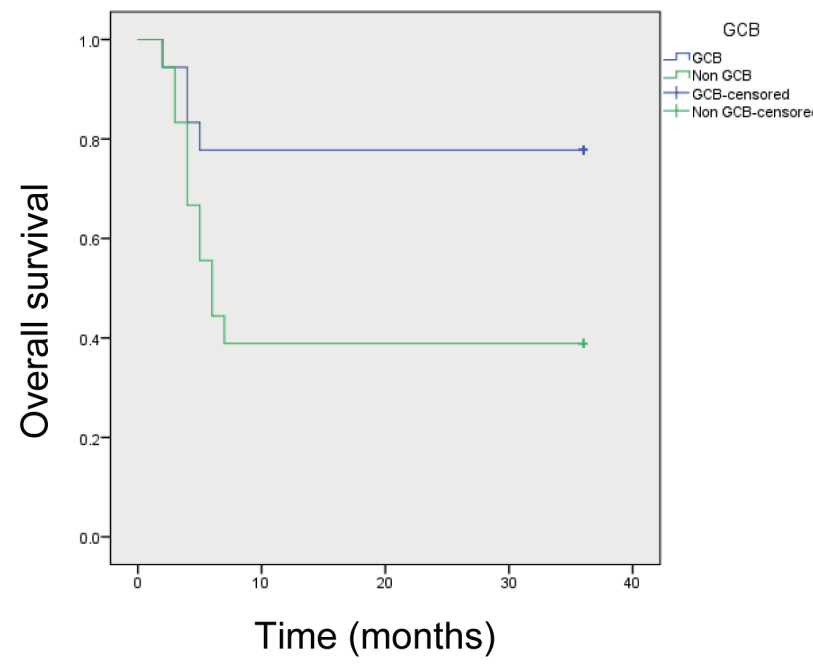

B

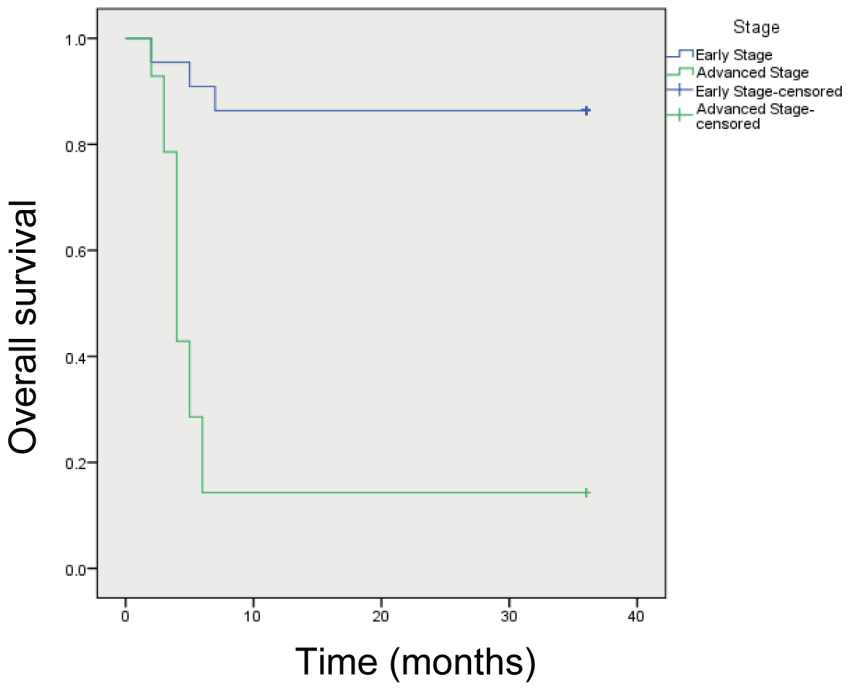

Fig. 2. Three-year overall survival of patients with DLBCL. A: Based on cell-origin subtype (GCB vs. non-GCB). B: Based on clinical stage (early vs. advanced).

studies indicated no significant differences between nodal/extra nodal location and cell-origin subtype of DLBCL. ${ }^{17-19}$

The results showed that the proportions of patients with GCB- and non-GCB-subtype DLBCL at Kariadi General Hospital Semarang are relatively equal (50\%); previous studies in Europe and the USA revealed a slightly higher proportion $(60 \%)$ of patients with GCB subtype DLBCL. Differences in frequency between GCB and non-GCB DLBCL are highly dependent on the geographical location, race, median age of the patient population and methodology used by researchers. However, the proportion of patients with non-GCB subtype DLBCL in Asian countries is generally lower than that of the rest of the world. This difference in proportion may be closely related to the characteristics of race and geographical location. These previous findings coincide with the result of the present study, where patients with GCB-subtype had a lower proportion $(50 \%)^{2}, 19,20$

Survival analysis by the log-rank test indicated that patients with non-GCB subtype DLBCL generally have significantly lower overall survival (38.9\%) compared with those with the GCB subtype (77.8\%). The median survival time of non-GCB DLBCL patients was 6 months. Patients with advanced-stage DLBCL also showed significantly lower overall survival (14.3\%) compared with those with early-stage DLBCL (86.4\%). The median survival time of patients with advanced-stage was 5 months (Table 3). Patients with 
Table 3. Overall survival of patients based on cell-origin subtype and disease stage

\begin{tabular}{lcc}
\hline & Number of events $(\%$ survival $)$ & $P$ \\
\hline All DLBCL patients $(n=36)$ & $15(58.3 \%)$ & 0.026 \\
GCB subtype & $4(77.8 \%)$ & \\
Non-GCB subtype & $11(38.9 \%)$ & $<0.001$ \\
\hline All DLBCL patients $(n=36)$ & $15(58.3 \%)$ & \\
Early $(n=22)$ & $3(86.4 \%)$ & \\
Advanced $(n=14)$ & $12(14.3 \%)$ & \\
\hline
\end{tabular}

GCB-subtype DLBCL are known to have a better prognosis and higher overall survival and progression free survival than those with the non-GCB subtype. Patients with GCB-subtype DLBCL had a 1-year overall survival rate of $90 \%$ and a 2 -year survival rate of $74 \%$. By comparison, patients with non-GCB-subtypes DLBCL had a 1 -year overall survival rate of $61 \%$ and a 2 -year survival rate of $46 \%$; these values are generally lower than those obtained for the GCB subtype. ${ }^{2,13,18}$ The present study obtained similar results, that is, patients with GCB-subtype DLBCL at Kariadi General Hospital have significantly better 2-year overall survival (77.8\%) compared with those with the non-GCB subtype (38.9\%). The overall survival of patients with GCB-subtype DLBCL is comparable with that of the same group in other studies (77.8\% vs. $74 \%)$, Among patients with GCB-subtype DLBCL who died in the first year of this study, four had an advanced tumor stage, and 2 showed MUM1 overexpression or was triple-positive for CD10, BCL6 and MUM1. Earlier research demonstrated that patients with GCB-subtype DLBCL and triple positive for CD10, BCL6 and MUM1 have lower overall survival compared with those with classic GCB-subtype DLBCL (i.e., positive CD10, positive BCL6 and negative MUM1). ${ }^{21,22}$

In this study, patients with non-GCB subtype DLBCL in Semarang had lower overall survival compared with those with non-GCB subtype DLBCL in other studies ( $38.9 \%$ vs. $46 \%$ ). The difference observed could be due to the large number of patients with nonGCB subtype DLBCL who died in the first year of the study; among the 12 patients who died, 9 (75\%) were at an advanced tumor stage. Patients with non-GCB subtypes DLBCL are known to have poor prognosis. The inferior prognosis of non-GCB subtype DLBCL is related to the presence of more mutations in non-GCB subtype DLBCL than in the GCB subtype. In the nonGCB subtype of DLBCL, mutations were found in at least 20 growth regulating genes, including BCL6, INK4, PRDM1, TNFAIP3, SPIB, CARD11, MYD88, MYC, BCL2, NFKB, CD79A, CD79B, CREBBP, E300,
MLL2, MEF2B, MEF2B, TBL1, NOTCH1, NOTCH2, BRAF, and TP53. By comparison, in the GCB subtypes of DLBCL, mutations were found in at least seven growth-regulating genes, namely, BCL2, EZH2, CREBBP, TNFRSF14, GNA13, SGK1, and C-REL. ${ }^{23-25}$

In conclusion, patients with non-GCB subtypes DLBCL have significantly lower 3-year overall survival than those with the GCB subtype (38.9\% vs. $77.8 \% ; P=$ 0.026). Patients with advanced stages of the disease also had significantly lower overall survival compared with those at early stages $(14.3 \%$ vs. $86.4 \% ; P<0.001)$.

Acknowledgments: This study was financially supported by a research grant from the Faculty of Medicine, Diponegoro University, Semarang, Central Java, Indonesia

The authors declare no conflict of interest.

\section{REFERENCES}

1 Globocan.iarc.fr. Cancer Fact Sheet [Internet]. Lyon; 2018 [cited 2019 Sep 9]. Available from: https://globocan.iarc.fr/

2 Swerdlow SH, Campo E, Pileri SA, Harris NL, Stein H, Siebert R, et al. The 2016 revision of the World Health Organization classification of lymphoid neoplasms. Blood. 2016;127:2375-90. DOI: 10.1182/blood-2016-01-643569, PMID: 26980727

3 Habara T, Sato Y, Takata K, Iwaki N, Okumura H, Sonobe H, et al. Germinal center B-cell-like versus non-germinal center B-cell-like as important prognostic factor for localized nodal DLBCL. J Clin Exp Hematop. 2012;52:91-9. DOI: 10.3960/ jslrt.52.91, PMID: 23037624

4 Syahrin RA, Ham MF, Harahap AS, Makes B, Hardjolukito ESR. Perbedaan Imunoekspresi Caspase-3 Antara Diffuse Large B-Cell Lymphoma Subtipe Germinal Center B-CellLike dan Non-Germinal Center B-Cell-Like. Indones J Pathol. 2018;27:1-6. Epub 2018 Feb 20.

5 Read JA, Koff JL, Nastoupil LJ, Williams JN, Cohen JB, Flowers CR. Evaluating cell-of-origin subtype methods for predicting diffuse large B-cell lymphoma survival: a meta-analysis of gene expression profiling and immunohistochemistry algorithms. Clin Lymphoma Myeloma Leuk. 2014;14:460-467.e2. DOI: 10.1016/j.clml.2014.05.002, PMID: 25052052

6 Testoni M, Zucca E, Young KH, Bertoni F. Genetic lesions in diffuse large B-cell lymphomas. Ann Oncol. 2015;26:106980. DOI: 10.1093/annonc/mdv019, PMID: 25605746 
7 Camicia R, Winkler HC, Hassa PO. Novel drug targets for personalized precision medicine in relapsed/refractory diffuse large B-cell lymphoma: a comprehensive review. Mol Cancer. 2015;14:207. DOI: 10.1186/s12943-015-0474-2, PMID: 26654227

8 Hans CP, Weisenburger DD, Greiner TC, Gascoyne RD, Delabie J, Ott G, et al. Confirmation of the molecular classification of diffuse large B-cell lymphoma by immunohistochemistry using a tissue microarray. Blood. 2004;103:275-82. DOI: 10.1182/blood-2003-05-1545, PMID: 14504078

9 Wang XJ, Seegmiller AC, Reddy NM, Li S. CD30 expression and its correlation with $M Y C$ rearrangement in de novo diffuse large B-cell lymphoma. Eur J Haematol. 2016;97:39-47. DOI: 10.1111/ejh.12680, PMID: 26340843

10 Yoo C, Kim S, Sohn BS, Kim JE, Yoon DH, Huh J, et al. Modified number of extranodal involved sites as a prognosticator in R-CHOP-treated patients with disseminated diffuse large B-cell lymphoma. Korean J Intern Med. 2010;25:301-8. DOI: 10.3904/kjim.2010.25.3.301, PMID: 20830228

11 Sehn LH, Gascoyne RD. Diffuse large B-cell lymphoma: optimizing outcome in the context of clinical and biologic heterogeneity. Blood. 2015;125:22-32. DOI: 10.1182/ blood-2014-05-577189, PMID: 25499448

12 Hwang HS, Yoon DH, Hong JY, Park CS, Lee YS, Ko YH, et al. The cell-of-origin classification of diffuse large B cell lymphoma in a Korean population by the Lymph2Cx assay and its correlation with immunohistochemical algorithms. Ann Hematol. 2018;97:2363-72. DOI: 10.1007/s00277-0183442-2, PMID: 30069703

13 Klapper W, Kreuz M, Kohler CW, Burkhardt B, Szczepanowski M, Salaverria I, et al.; Molecular Mechanisms in Malignant Lymphomas Network Project of the Deutsche Krebshilfe. Patient age at diagnosis is associated with the molecular characteristics of diffuse large B-cell lymphoma. Blood. 2012;119:1882-7. DOI: 10.1182/blood-2011-10-388470, PMID: 22238326

14 Zhou Z, Sehn LH, Rademaker AW, Gordon LI, LaCasce AS, Crosby-Thompson A, et al. An enhanced International Prognostic Index (NCCN-IPI) for patients with diffuse large B-cell lymphoma treated in the rituximab era. Blood. 2014;123:83742. DOI: 10.1182/blood-2013-09-524108, PMID: 24264230

15 Li S, Young KH, Medeiros LJ. Diffuse large B-cell lymphoma. Pathology. 2018;50:74-87. DOI: 10.1016/ j.pathol.2017.09.006, PMID: 29167021

16 Bruno Ventre M, Ferreri AJM, Gospodarowicz M, Govi S, Messina C, Porter D, et al.; International Extranodal Lymphoma Study Group. Clinical features, management, and prognosis of an international series of 161 patients with limited-stage diffuse large B-cell lymphoma of the bone (the IELSG-14 study). Oncologist. 2014;19:291-8. DOI: 10.1634/ theoncologist.2013-0249, PMID: 24567283
17 Gogia A, Das CK, Kumar L, Sharma A, Tiwari A, Sharma $\mathrm{MC}$, et al. Diffuse large B-cell lymphoma: an institutional analysis. South Asian J Cancer. 2018;07:200-2. DOI: 10.4103/ sajc.sajc 65 18, PMID: 30112341

18 Abdelhamid T, Samra M, Ramadan H, Mehessin M, Mokhtar N. Clinical prognostic factors of diffuse large B cell nonHodgkin lymphoma: A retrospective study. J Egypt Natl Canc Inst. 2011;23:17-24. DOI: 10.1016/j.jnci.2011.07.003, PMID: 22099932

19 Salles G, de Jong D, Xie W, Rosenwald A, Chhanabhai M, Gaulard P, et al. Prognostic significance of immunohistochemical biomarkers in diffuse large B-cell lymphoma: a study from the Lunenburg Lymphoma Biomarker Consortium. Blood. 2011;117:7070-8. DOI: 10.1182/ blood-2011-04-345256, PMID: 21536860

20 Hwang HS, Park CS, Yoon DH, Suh C, Huh J. High concordance of gene expression profiling-correlated immunohistochemistry algorithms in diffuse large B-cell lymphoma, not otherwise specified. Am J Surg Pathol. 2014;38:1046-57. DOI: 10.1097/PAS.0000000000000211, PMID: 24705314

21 Li T, Medeiros LJ, Lin P, Yin H, Littlejohn M, Im W, et al. Immunohistochemical profile and fluorescence in situ hybridization analysis of diffuse large B-cell lymphoma in northern China. Arch Pathol Lab Med. 2010;134:759-65. DOI: 10.5858/134.5.759, PMID: 20441508

22 Lu TX, Miao Y, Wu JZ, Gong QX, Liang JH, Wang Z, et al. The distinct clinical features and prognosis of the CD10+MUM1+ and CD10-Bc16-MUM1- diffuse large B-cell lymphoma. Sci Rep. 2016;6:20465. DOI: 10.1038/ srep20465, PMID: 26857366

23 Rosenwald A, Wright G, Chan WC, Connors JM, Campo E, Fisher RI, et al.; Lymphoma/Leukemia Molecular Profiling Project. The use of molecular profiling to predict survival after chemotherapy for diffuse large-B-cell lymphoma. N Engl J Med. 2002;346:1937-47. DOI: 10.4149/neo_2013_010, PMID: 23067219

24 Lohr JG, Stojanov P, Lawrence MS, Auclair D, Chapuy B, Sougnez C, et al. Discovery and prioritization of somatic mutations in diffuse large B-cell lymphoma (DLBCL) by wholeexome sequencing. Proc Natl Acad Sci USA. 2012;109:387984. DOI: 10.1073/pnas.1121343109, PMID: 22343534

25 Rossi D, Ciardullo C, Gaidano G. Genetic aberrations of signaling pathways in lymphomagenesis: revelations from next generation sequencing studies. Semin Cancer Biol. 2013;23:422-30. DOI: 10.1016/j.semcancer.2013.04.002, PMID: 23665546 